University of Nebraska - Lincoln

DigitalCommons@University of Nebraska - Lincoln

January 1987

\title{
Atomic hydrogen in a uniform magnetic field: Low-lying energy levels for fields above $10^{9} \mathrm{G}$
}

Chih-Ray Liu

University of Nebraska - Lincoln

Anthony F. Starace

University of Nebraska-Lincoln, astarace1@unl.edu

Follow this and additional works at: https://digitalcommons.unl.edu/physicsstarace

Part of the Physics Commons

Liu, Chih-Ray and Starace, Anthony F., " Atomic hydrogen in a uniform magnetic field: Low-lying energy levels for fields above $10^{9} \mathrm{G}$ " (1987). Anthony F. Starace Publications. 30.

https://digitalcommons.unl.edu/physicsstarace/30

This Article is brought to you for free and open access by the Research Papers in Physics and Astronomy at DigitalCommons@University of Nebraska - Lincoln. It has been accepted for inclusion in Anthony F. Starace Publications by an authorized administrator of DigitalCommons@University of Nebraska - Lincoln. 


\title{
Atomic hydrogen in a uniform magnetic field: Low-lying energy levels for fields above $10^{9} \mathrm{G}$
}

\author{
Chih-Ray Liu and Anthony F. Starace \\ Department of Physics and Astronomy, The University of Nebraska, Lincoln, Nebraska 68588-0111
}

(Received 14 July 1986)

\begin{abstract}
The energies and wave functions of the $1 s, 2 s, 2 p\left(m_{l}=0\right)$, and $2 p\left(m_{l}= \pm 1\right)$ levels of hydrogen in a uniform magnetic field $B\left(10^{9} \mathrm{G} \leqq B \leqq 10^{12} \mathrm{G}\right)$ are calculated in two cylindrical adiabatic approximations, each of which includes the influence of the Coulomb field on the radial motion of the electron. For the lowest levels of each symmetry [i.e., the $1 s, 2 p\left(m_{l}=0\right)$ and $2 p\left(m_{l}= \pm 1\right)$ levels] our calculations provide rigorously both upper and lower bounds on the true level energies and binding energies. We present these cylindrical adiabatic upper and lower bounds on the binding energies together with the spherical adiabatic upper and lower bounds of Starace and Webster as well as all superior variational lower bounds known to us in order to provide in one place stringent tests of past (and future) calculations. The very detailed 1984 eigenfunction expansion results of Rösner, Wunner, Herold, and Ruder are found to be consistent with the upper and lower bounds presented.
\end{abstract}

\section{INTRODUCTION}

The nonseparability of the Schrödinger equation for an electron in combined Coulomb and uniform magnetic fields makes the theoretical description of even so simple a system as a hydrogen atom in a uniform magnetic field quite difficult when the two field strengths are comparable. The intrinsic theoretical interest of such a problem together with the obvious applications to astrophysical phenomena have generated a great deal of interest in the subject, which has been reviewed elsewhere. ${ }^{1-4}$ In particular, most recently there has been a veritable explosion of interest in the accurate theoretical calculation of the binding energies for the low-lying levels of atomic hydrogen in the case of magnetic field strengths comparable to and greater than that of the Coulomb field. This case has been seen as a challenge to newer methods of perturbation theory ${ }^{5-18}$ as well as for variational approaches ${ }^{19-24}$ which incorporate both the spherical and the cylindrical symmetries inherent in the system.

In the present paper we present adiabatic approximation results in cylindrical coordinates for the lowest energy levels in atomic hydrogen, primarily for magnetic fields above $10^{9} \mathrm{G}$. This work complements the adiabatic approximation results in spherical coordinates of Starace and Webster, ${ }^{25}$ who were concerned primarily with magnetic fields below $10^{9} \mathrm{G}$. As was the case in spherical coordinates, ${ }^{25}$ our adiabatic approximations provide both upper and lower bounds on the binding energies of the lowest levels in hydrogen for each symmetry. Our rigorous upper bounds together with either our rigorous lower bounds or those of the best variational calculations provide stringent tests of previous (and future) calculations for very high magnetic field strengths. While for the highest field strengths considered our results are comparable or superior to perturbation calculations employing many configurations or to variational calculations employing several parameters, nevertheless our adiabatic calculations employ only a single configuration wave function and no parameters.
While there have been several previous adiabatic calculations in cylindrical coordinates, ${ }^{5,26-31}$ all but two of them ${ }^{28,29}$ ignore the effect of the Coulomb field in the radial direction perpendicular to the magnetic field. In addition to treating the effect of the Coulomb field in the radial direction, we also consider formally the coupling matrix elements between adiabatic channels. We calculate explicitly the diagonal coupling matrix element within each adiabatic channel. Omitting or including this matrix element in our calculations provides, respectively, rigorous lower or upper bounds on the lowest energy level for each symmetry state of the system.

In Sec. II we present an exact formulation of the coupled adiabatic equations in cylindrical coordinates for the case of an electron in combined Coulomb and uniform magnetic fields. In Sec. III we present the two adiabatic approximations which are used in our calculations. Section IV presents calculated adiabatic upper and lower bounds on the binding energies of the $1 s, 2 p\left(m_{l}= \pm 1\right)$, and $2 p\left(m_{l}=0\right)$ levels as well as adiabatic approximation results for the $2 s$ level. It also contains our plots of wave-function intensities as a function of magnetic field strength and our conclusions on the comparison of our results with those of others. A preliminary report of this work has been presented elsewhere. ${ }^{32}$

\section{EXACT FORMULATION}

\section{A. Schrödinger equation}

The spin-independent Schrödinger equation in cylindrical coordinates for an electron in combined Coulomb and uniform magnetic fields is ${ }^{33}$

$$
\begin{aligned}
\left(\frac{-\hbar^{2}}{2 m_{e}} \nabla^{2}+\frac{|e| B}{2 m_{e} c} L_{z}\right. & +\frac{e^{2}}{8 m_{e} c^{2}} B^{2} \rho^{2} \\
& \left.-\frac{e^{2}}{\left(\rho^{2}+z^{2}\right)^{1 / 2}}\right) \psi(\mathbf{r})=E \psi(\mathbf{r}),
\end{aligned}
$$


where the magnetic field $B$ has been oriented along the $z$ axis, $L_{z}$ is the $z$ component of the orbital angular momentum operator, and $m_{e}$ is the electron mass. The operators in large parentheses are those for the kinetic energy, the linear Zeeman shift, the quadratic Zeeman shift, and the Coulomb energy. We shall use atomic units henceforth (i.e., $m_{e}=e=\hbar=1$ ) and introduce the strength parameter $\alpha$,

$$
\alpha \equiv B / 2 c=\left(2.12715 \times 10^{-10} \text { a.u. } / \mathrm{G}\right) B(\mathrm{G}) .
$$

Equation (1) in atomic units thus becomes

$$
\left[-\frac{1}{2} \nabla^{2}+\alpha L_{z}+\frac{1}{2} \alpha^{2} \rho^{2}-\left(\rho^{2}+z^{2}\right)^{-1 / 2}\right] \psi(\mathbf{r})=E \psi(\mathbf{r}) .
$$

In order to compare our results with those of nearly all other researchers, we have assumed in Eqs. (1) and (3) that the nucleus has infinite mass. Another reason for this assumption is that so-called $Z$ scaling of our results for hydrogen to other hydrogenic systems is only possible in the infinite mass case. ${ }^{2,34}$ For the case considered here, i.e., neutral hydrogen atoms at rest in a magnetic field, our infinite mass results may be transformed rigorously to finite mass results. ${ }^{35}$

Taking account of the azimuthal symmetry of the problem, we substitute

$$
\psi(\mathbf{r})=G(\rho, z) e^{i m \phi}(2 \pi \rho)^{-1 / 2}
$$

into Eq. (3) to get the following equation for $G(\rho, z)$ :

$$
\begin{aligned}
& \int \frac{\partial^{2}}{\partial \rho^{2}}+\frac{\partial^{2}}{\partial z^{2}}-\frac{\left(m^{2}-1 / 4\right)}{\rho^{2}}+\frac{2}{\left(\rho^{2}+z^{2}\right)^{1 / 2}} \\
& \left.-\alpha^{2} \rho^{2}+2 E^{\prime}\right] G(\rho, z)=0 \text {. }
\end{aligned}
$$

In Eq. (5) the energy $E^{\prime}$ is defined by

$$
E^{\prime} \equiv E-\alpha m \text {. }
$$

\section{B. Expansion in adiabatic radial functions}

The $\rho$-dependent terms in Eq. (5) may be used to generate a complete set of radial functions, as follows:

$$
\begin{array}{r}
\left(\frac{d^{2}}{d \rho^{2}}-\frac{\left(m^{2}-1 / 4\right)}{\rho^{2}}+\frac{2}{\left(\rho^{2}+z^{2}\right)^{1 / 2}}-\alpha^{2} \rho^{2}\right) f_{n m}(z ; \rho) \\
=-2 W_{n m}(z) f_{n m}(z ; \rho) .
\end{array}
$$

In Eq. (7), the coordinate $z$ is treated as a parameter and the eigenvalue $W_{n m}(z)$ is a $z$-dependent potential. Both the potential $W_{n m}(z)$ and the radial function $f_{n m}(z ; \rho)$ are indexed sequentially by the integer $n(\geqq 1)$ as well as by the magnetic quantum number $m$. Because Eq. (7) depends on $m$ only through $m^{2}$, both $W_{n m}$ and $f_{n m}$ depend only on $|m|$. We normalize the radial functions according to

$$
\int_{0}^{\infty} d \rho f_{n m}(z ; \rho) f_{n^{\prime} m}(z ; \rho)=\delta_{n n^{\prime}} \text {. }
$$

The solution, $G(\rho, z)$, to Eq. (5) may be expanded in terms of the adiabatic radial functions $f_{n m}$ as follows:

$$
G(\rho, z)=\sum_{n^{\prime}} f_{n^{\prime} m}(z ; \rho) q_{n^{\prime} m v \pi}(z) .
$$

In Eq. (9) we have introduced the $z$-dependent functions $q_{n^{\prime} m v \pi}$, where $n^{\prime}$ denotes the index of the adiabatic radial function $f_{n^{\prime} m}, m$ denotes the magnetic quantum number, $v$ is an integer (for bound states) denoting the energy eigenvalue, $E_{v}^{\prime}$, and $\pi$ denotes the reflection parity with respect to the $x-y$ plane of the function $G(\rho, z)$. Since $f_{n^{\prime} m}(z ; \rho)$ is symmetric in $z$, this reflection symmetry is carried by the function $q$. Note that the total parity $P$ of the wave function $\psi(\rho, z, \phi)$ defined in Eq. (4) is given by

$$
P=\pi(-1)^{m} \text {. }
$$

Substituting Eq. (9) in Eq. (5), multiplying from the left by $f_{n m}(z ; \rho)$, integrating over $\rho$, and using Eqs. (7) and (8), gives the following set of coupled equations for the functions $q$ :

$$
\begin{aligned}
& {\left[\frac{d^{2}}{d z^{2}}+2 E_{v}^{\prime}-2 W_{n m}(z)\right] q_{n m v \pi}(z)} \\
& +2 \sum_{n^{\prime}}\left[f_{n m}, \frac{\partial f_{n^{\prime} m}}{\partial z}\right] \frac{\partial q_{n^{\prime} m v \pi}(z)}{\partial z} \\
& +\sum_{n^{\prime}}\left[f_{n m}, \frac{\partial^{2} f_{n^{\prime} m}}{\partial z^{2}}\right] q_{n^{\prime} m v \pi}(z)=0,
\end{aligned}
$$

where we have used the following notation (with $s=1$ or 2) for the coupling matrix elements:

$$
\left[f_{n m}, \frac{\partial^{s} f_{n^{\prime} m}}{\partial z^{s}}\right] \equiv \int_{0}^{\infty} d \rho f_{n m}(z ; \rho) \frac{\partial^{s} f_{n^{\prime} m}}{\partial z^{s}}(z ; \rho) \text {. }
$$

\section{Properties of the coupling matrix elements}

Differentiation of the orthonormality equation (8) with respect to $z$ shows that the first derivative matrix elements in Eq. (12) are antisymmetric,

$$
\left[f_{n m}, \frac{\partial f_{n^{\prime} m}}{\partial z}\right]=-\left(\frac{\partial f_{n m}}{\partial z}, f_{n^{\prime} m}\right) \text {. }
$$

This implies that the diagonal matrix elements vanish,

$$
\left[f_{n m}, \frac{\partial f_{n m}}{\partial z}\right]=0 \text {. }
$$

Similarly, differentiation of the first-derivative coupling matrix element [Eq. (12) with $s=1$ ] with respect to $z$ results in the following expression for the second-derivative coupling matrix element [Eq. (12) with $s=2$ ]:

$$
\left[f_{n m}, \frac{\partial^{2} f_{n^{\prime} m}}{\partial z^{2}}\right)=-\left(\frac{\partial f_{n m}}{\partial z}, \frac{\partial f_{n^{\prime} m}}{\partial z}\right)+\frac{d}{d z}\left[f_{n m}, \frac{\partial f_{n^{\prime} m}}{\partial z}\right] .
$$

The diagonal matrix element is thus seen to be negative definite,

$$
\left[f_{n m}, \frac{\partial^{2} f_{n, m}}{\partial z^{2}}\right]=-\left[\frac{\partial f_{n m}}{\partial z}, \frac{\partial f_{n m}}{\partial z}\right) \leqq 0
$$




\section{ADIABATIC APPROXIMATIONS}

Solution of the infinite set of coupled differential equations (11) constitutes an exact solution to the problem of an electron in combined Coulomb and uniform magnetic fields. Practically, of course, one obtains an approximate solution by truncating the infinite set of coupled equations to some finite set. In other words, one ignores some of the coupling matrix elements defined in Eq. (12). Ordinarily one must justify this truncation by a detailed consideration of the magnitudes of the coupling matrix elements. One may show, however, that the two adiabatic approximations defined below provide rigorous lower and upper bounds to the system's lowest energy level of each symmetry. ${ }^{36}$ (The symmetry of the system is defined by the magnetic quantum number $m$ and by the parity $P$ or, equivalently, the reflection parity $\pi$ [cf. Eq. (10)].) Thus the energy levels (or, equivalently, the binding energies) calculated by the adiabatic approximations below are meaningful results even without a detailed analysis of the strength of the coupling matrix elements. The hydrogenic designations of the energy levels we have calculated by solving the adiabatic equations below are listed in Table I.

\section{A. Lower-bound adiabatic approximation}

If we neglect all coupling matrix elements in Eq. (11), we obtain

$$
\left[-\frac{1}{2} \frac{d^{2}}{d z^{2}}+W_{n m}(z)\right] q_{n m v \pi}(z)=E_{v}^{\prime} q_{n m v \pi}(z) .
$$

Solution of Eq. (17) for fixed $n$ and $m$ gives a spectrum of eigenvalues $E_{v}^{\prime}$ and their corresponding eigenfunctions $q_{n m v \pi}$. One may prove ${ }^{36}$ that for a value of $n$ appropriate to the lowest energy level for a given $m$ and $\pi$, the lowest energy $E_{v}^{\prime}$ computed from Eq. (17) is a lower bound on the lowest exact energy for the same $m$ and $\pi$. Equation (17) has been studied by Baldereschi and Bassani ${ }^{28}$ and by Tanaka and Shinada. ${ }^{29}$

\section{B. Upper-bound adiabatic approximation}

If we neglect all off-diagonal coupling matrix elements in Eq. (11), we obtain

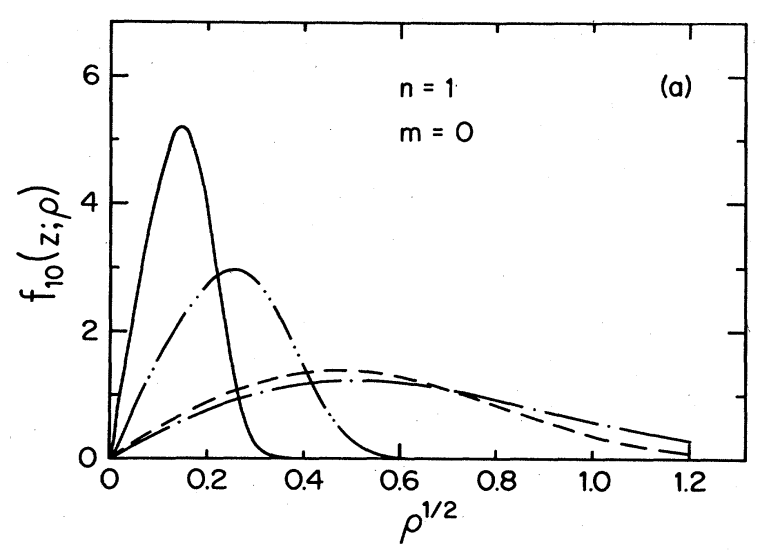

TABLE I. Hydrogenic designations and threshold shifts for calculated energy levels.

\begin{tabular}{|c|c|c|c|c|c|c|}
\hline$n$ & $m$ & $v$ & $\pi$ & $\begin{aligned} & \text { Parity } \\
= & \pi(-1)^{m}\end{aligned}$ & $\begin{array}{l}\text { Hydrogenic } \\
\text { designation }^{\mathrm{a}}\end{array}$ & $\begin{array}{l}\text { Threshold shift } \\
\alpha(n+|m|+m)\end{array}$ \\
\hline 1 & 0 & 1 & + & + & $1 s\left(m_{l}=0\right)$ & $\alpha$ \\
\hline 1 & 0 & 2 & + & + & $2 s\left(m_{l}=0\right)$ & $\alpha$ \\
\hline 1 & 0 & 1 & - & - & $2 p\left(m_{l}=0\right)$ & $\alpha$ \\
\hline 1 & +1 & 1 & + & - & $2 p\left(m_{l}=+1\right)$ & $3 \alpha$ \\
\hline 1 & -1 & 1 & + & - & $2 p\left(m_{l}=-1\right)$ & $\alpha$ \\
\hline
\end{tabular}

${ }^{a}$ Appropriate in the limit of zero magnetic field.

$$
\begin{array}{r}
{\left[-\frac{1}{2} \frac{d^{2}}{d z^{2}}+W_{n m}(z)-\frac{1}{2}\left[f_{n m}, \frac{\partial^{2} f_{n m}}{\partial z^{2}}\right]\right] q_{n m v \pi}(z)} \\
=E_{v}^{\prime} q_{n m v \pi}(z)
\end{array}
$$

Equation (18) differs from Eq. (17) by the inclusion of the diagonal second-derivative coupling matrix element, which from Eq. (16) is seen to contribute a positivedefinite upward shift to the energy spectrum. One may prove $^{36}$ that for a value of $n$ appropriate to the lowest energy level for a given $m$ and $\pi$, the lowest energy $E_{v}^{\prime}$ computed from Eq. (18) is an upper bound on the lowest exact energy for the same $m$ and $\pi$.

\section{RESULTS AND DISCUSSION}

We have solved Eqs. (7), (17), and (18) for the wave functions, potentials, and low-lying energies of a hydrogen atom in a uniform magnetic field for field strengths $0.05 \leqq \alpha \leqq 1000$ corresponding to magnetic fields in the range $0.235 \times 10^{9} \mathrm{G} \leqq B \leqq 4.70 \times 10^{12} \mathrm{G}$. We present here our detailed results for the wave functions, potentials, and binding energies of the $1 s, 2 s$, and $2 p$ levels of hydrogen. Our rigorous upper and lower bounds for the $1 s$ and $2 p$ binding energies provide very stringent tests of past and future calculations for these levels.

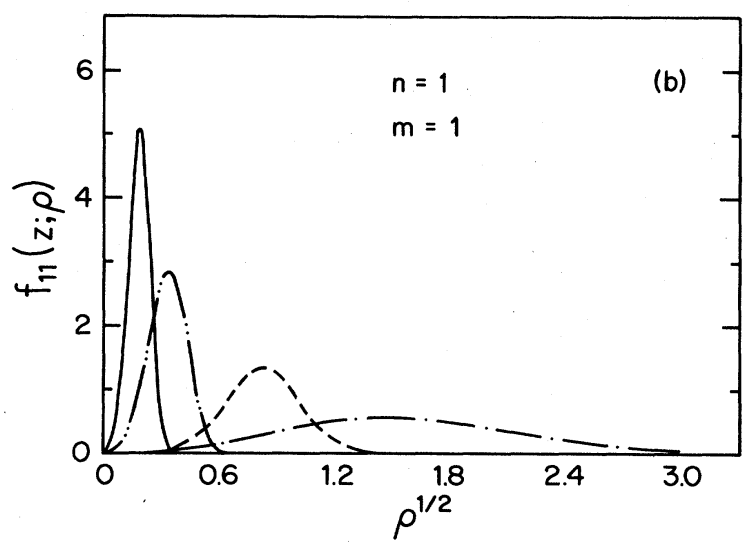

FIG. 1. Plots of the lowest (i.e., $n=1)$ functions $f_{n m}(z ; \rho)$ as a function of $\rho^{1 / 2}$ for $z=0$. Functions with magnetic quantum number $m=0$ are shown in (a) and those with $m=1$ are shown in (b). Results for the following four values of the magnetic field strength parameter are shown: dash-dot curves, $\alpha=0.05$; dashed curves, $\alpha=2.5$; dash-double-dot curves, $\alpha=100$; solid curves, $\alpha=1000$. 


\section{A. Wave functions and potentials}

The lowest (i.e., $n=1)$ radial functions $f_{n m}(z ; \rho)$ obtained as the solution of Eq. (7) (for $m=0$ and 1 and $z=0$ ) are shown in Fig. 1 for the four magnetic field values corresponding to $\alpha=0.05,2.5,100$, and 1000. One observes very clearly the contraction of $f_{n m}$ toward lower values of $\rho$ as $\alpha$ increases. This is due to the increasing magnitude of the quadratic Zeeman term in Eq. (7). One observes as well that the $m=1$ functions have a greater radial extent than the $m=0$ functions due to the orbital angular momentum centrifugal barrier.

The eigenvalues of Eq. (7), $W_{n m}(z)$, which are the potentials used in Eqs. (17) and (18), are shown in Fig. 2 for $n=1$ and $m=0$ and 1 . In order to compare these potentials easily for different values of the magnetic field, each is plotted with respect to its asymptotic value, $W_{n m}(z \rightarrow \infty)=\alpha(n+|m|)$, which is labeled as zero in the figure. (See the discussion of threshold energy shifts in Sec. IV B below.) By thus removing this threshold energy shift, we see clearly that $W_{n m}(z)$ is quite flat and only weakly dependent on the magnetic field for $|z| \geqq 1$. For $|z| \approx 0$, on the other hand, $W_{n m}(z)$ becomes increasingly negative as the magnetic field strength increases. Comparison of Figs. 2(a) and 2(b) shows the effect of the magnetic quantum number, $m$ : for $m=1$ the potentials $W_{n m}(z)$ are much shallower than those for $m=0$ in the region $|z| \approx 0$.

The $z$-dependent wave functions $q_{n m v \pi}(z)$ obtained from Eq. (18), are shown in Figs. 3(a)-3(d) for the states corresponding to the hydrogen $1 s, 2 s, 2 p\left(m_{l}=0\right)$, and $2 p\left(m_{l}= \pm 1\right)$ states, respectively. Table I shows the correspondence between the quantum numbers $n m v \pi$ and the hydrogenic designations applicable for zero magnetic field. As was the case for the radial functions $f_{n m}(z ; \rho)$, one sees here that for increasing magnetic field the wave functions $q_{n m v \pi}(z)$ move closer to $|z| \approx 0$. Because of this contraction in $z$, the amplitude of the major antinode of $q_{n m v \pi}(z)$ increases in each case. This increase in amplitude is most dramatic for the two $v^{\pi}=1^{+}$states in Figs. $3(\mathrm{a})$ and $3(\mathrm{~d})$, which have only a single antinode centered about $z=0$.

The adiabatic approximation to $G(\rho, z)$, defined in Eq. (4), consists of setting $G(\rho, z)$ equal to a single term in the sum in Eq. (9), i.e.,

$$
G(\rho, z) \approx f_{n m}(z ; \rho) q_{n m v \pi}(z),
$$

where $f_{n m}(z ; \rho)$ is the solution of Eq. (7) and $q_{n m v \pi}(z)$ is obtained from either Eq. (17) or Eq. (18). In Figs. 4-7 we show this adiabatic approximation to $|G(\rho, z)|^{2}$ using functions $q_{n m v \pi}(z)$ calculated from Eq. (18) for the $1 s, 2 s$, $2 p\left(m_{l}=0\right)$, and $2 p\left(m_{l}= \pm 1\right)$ states of hydrogen. The $n m v \pi$ quantum numbers for these states are given in Table I. Each figure shows how the squared absoluteprobability amplitude contracts toward the origin with increasing magnetic field. Note that to show the probability distribution clearly, parts (a) and (b) of each figure are plotted on a different scale from parts (c) and (d). Note also the increasing similarity of the probabilities for the $1 s$ and $2 p\left(m_{l}= \pm 1\right)$ states with increasing magnetic field; these states differ only in the $m_{l}$ quantum number.

\section{B. Binding energies}

The binding energy of an electron in a Coulomb potential is altered in the presence of a uniform magnetic field. By solving Eq. (7) in the limit that $z \rightarrow \infty$, we find

$$
W_{n m}(z) \underset{z \rightarrow \infty}{\rightarrow} \alpha(n+|m|) .
$$

Substituting Eq. (20) into Eq. (17) and using Eq. (6) we obtain the asymptotic limit of Eq. (17),

$$
\left[-\frac{1}{2} \frac{d^{2}}{d z^{2}}+\alpha(n+|m|+m)\right] q_{n m v \pi}(z)=E_{v} q_{n m v \pi} .
$$

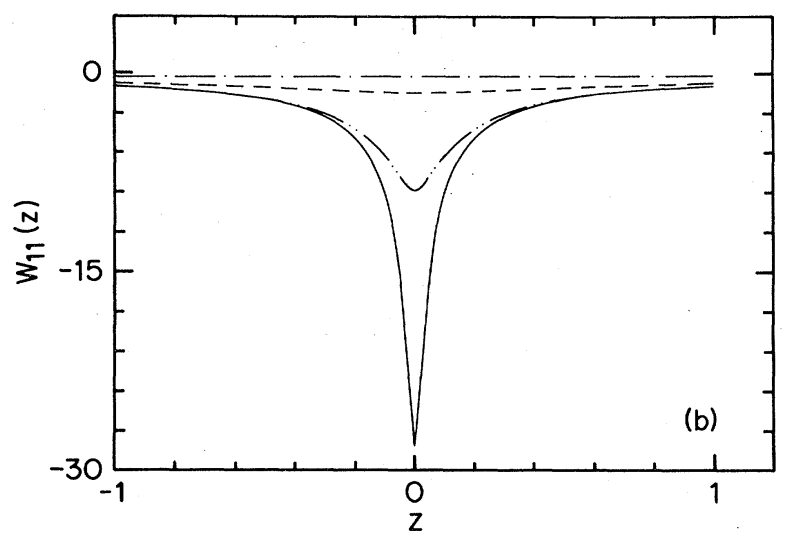

FIG. 2. Plots of the potentials $W_{n m}(z)$ as a function of $z$ relative to the asymptotic value $W_{n m}(z \rightarrow \infty)=\alpha(n+|m|)$, which is here set equal to zero for ease of comparison of results for different values of $\alpha$. Potentials with $n=1$ and $m=0$ are shown in (a) and those with $n=1$ and $m=1$ are shown in (b). Results for the following four values of the magnetic field strength parameter are shown: dash-dot curves, $\alpha=0.05$; dashed curves, $\alpha=2.5$; dash-double-dot curves, $\alpha=100$; solid curves, $\alpha=1000$. 

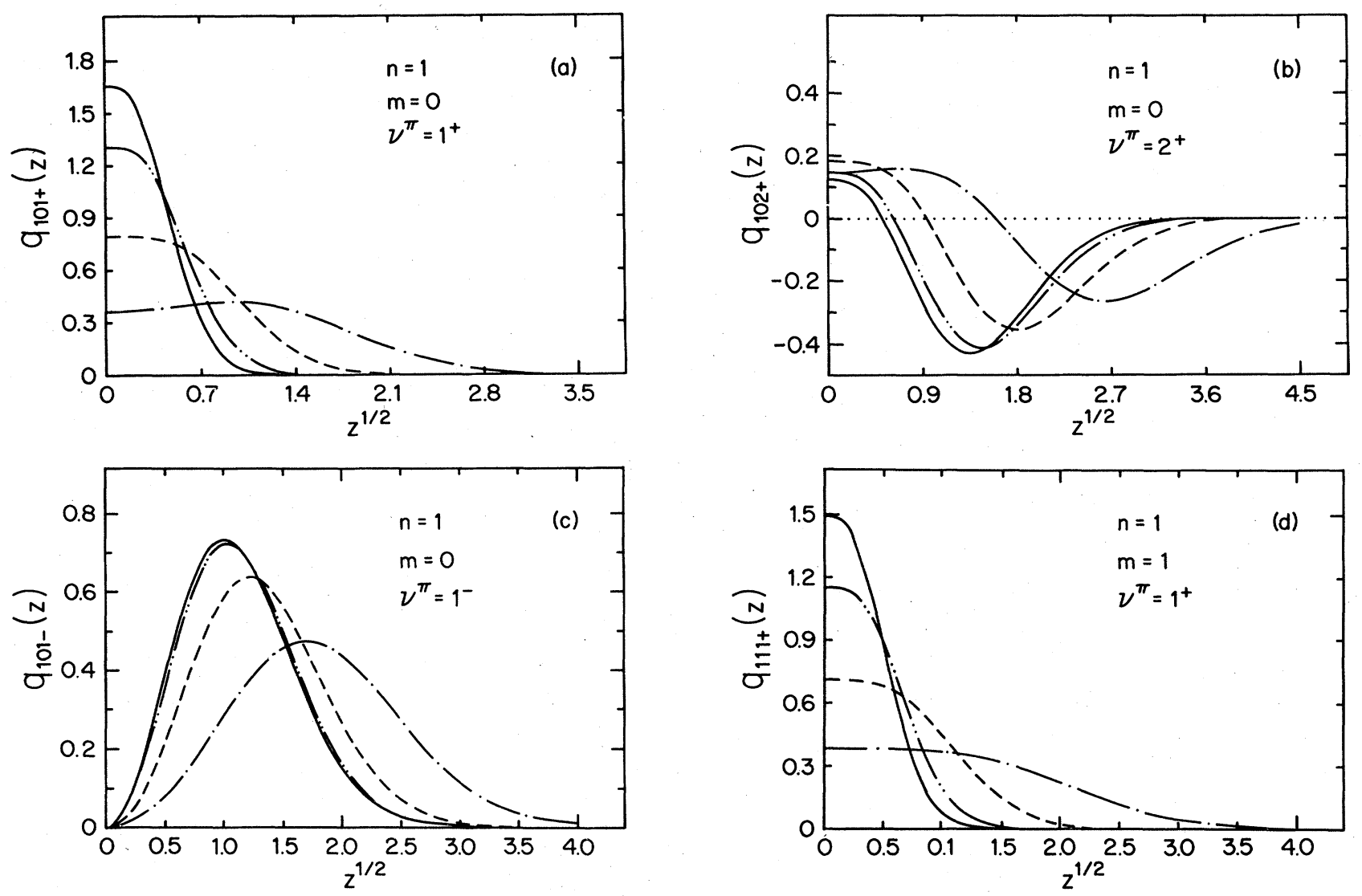

FIG. 3. Plots vs $z^{1 / 2}$ of the $z$-dependent wave functions $q_{n m v \pi}(z)$ obtained from Eq. (18) corresponding to the following four hydrogen states: (a) $1 s\left(m_{l}=0\right)$; (b) $2 s\left(m_{l}=0\right)$, (c) $2 p\left(m_{l}=0\right)$; and (d) $2 p\left(m_{l}= \pm 1\right)$. The quantum numbers $n m v \pi$ are shown in each figure and are defined in the text. Results are presented in each instance for the following four values of the magnetic field strength parameter: dash-dot curves, $\alpha=0.05$; dashed curves, $\alpha=2.5$; dash-double-dot curves, $\alpha=100$; solid curves, $\alpha=1000$.
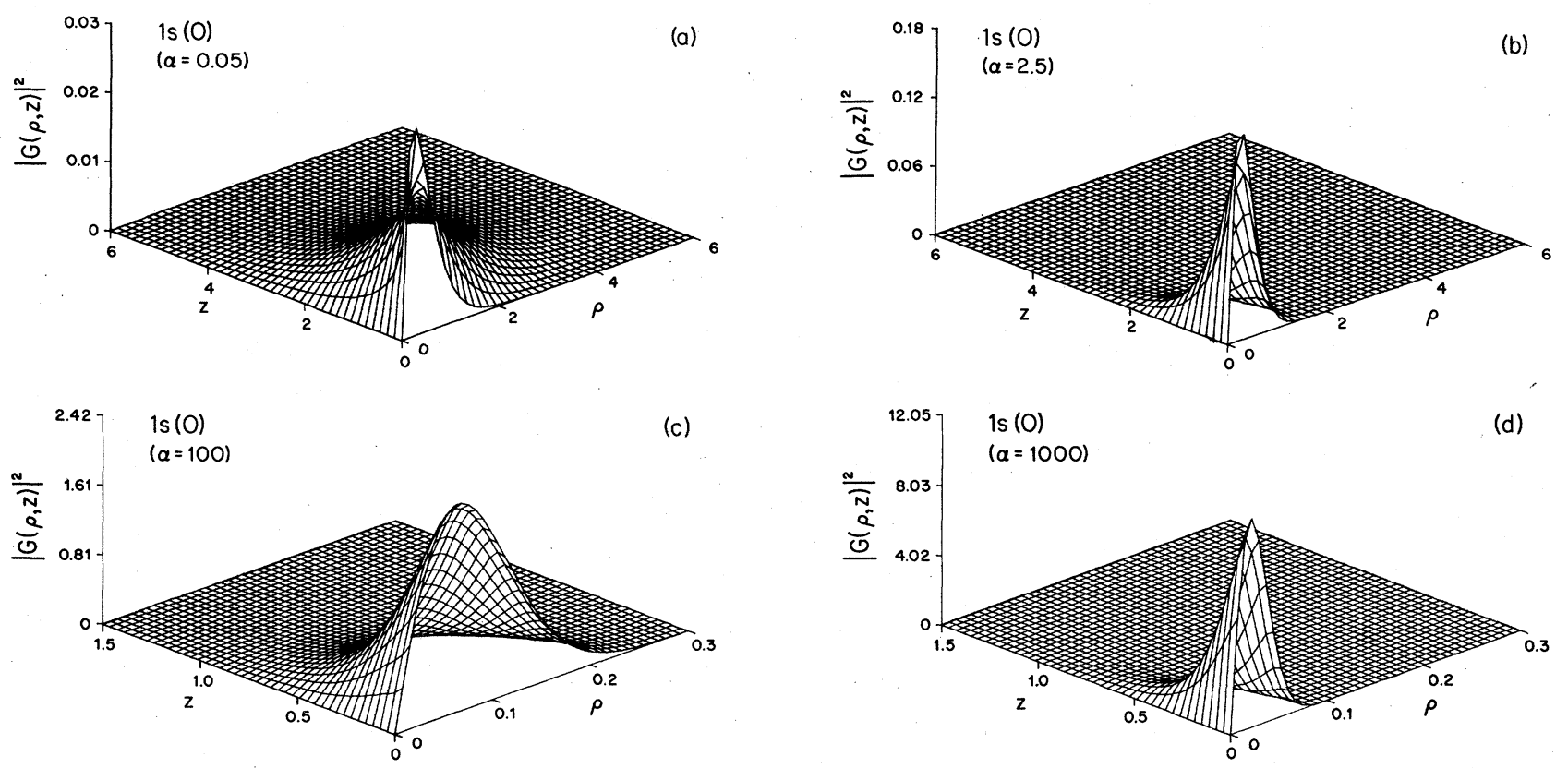

FIG. 4. Absolute square of the adiabatic approximation to the probability amplitude $G(\rho, z)$ [cf. Eq. (19)] for $n m v \pi \equiv 101+$ corresponding to the $1 s m_{l}=0$ level of hydrogen for four values of the magnetic field strength parameter $\alpha$ [cf. Eq. (2)]. 



FIG. 5. Absolute square of the adiabatic approximation to the probability amplitude $G(p, z)$ [cf. Eq. (19)] for $n m v \pi \equiv 102+$ corresponding to the $2 s\left(m_{l}=0\right)$ level of hydrogen for four values of the magnetic field strength parameter $\alpha$ [cf. Eq. (2)].
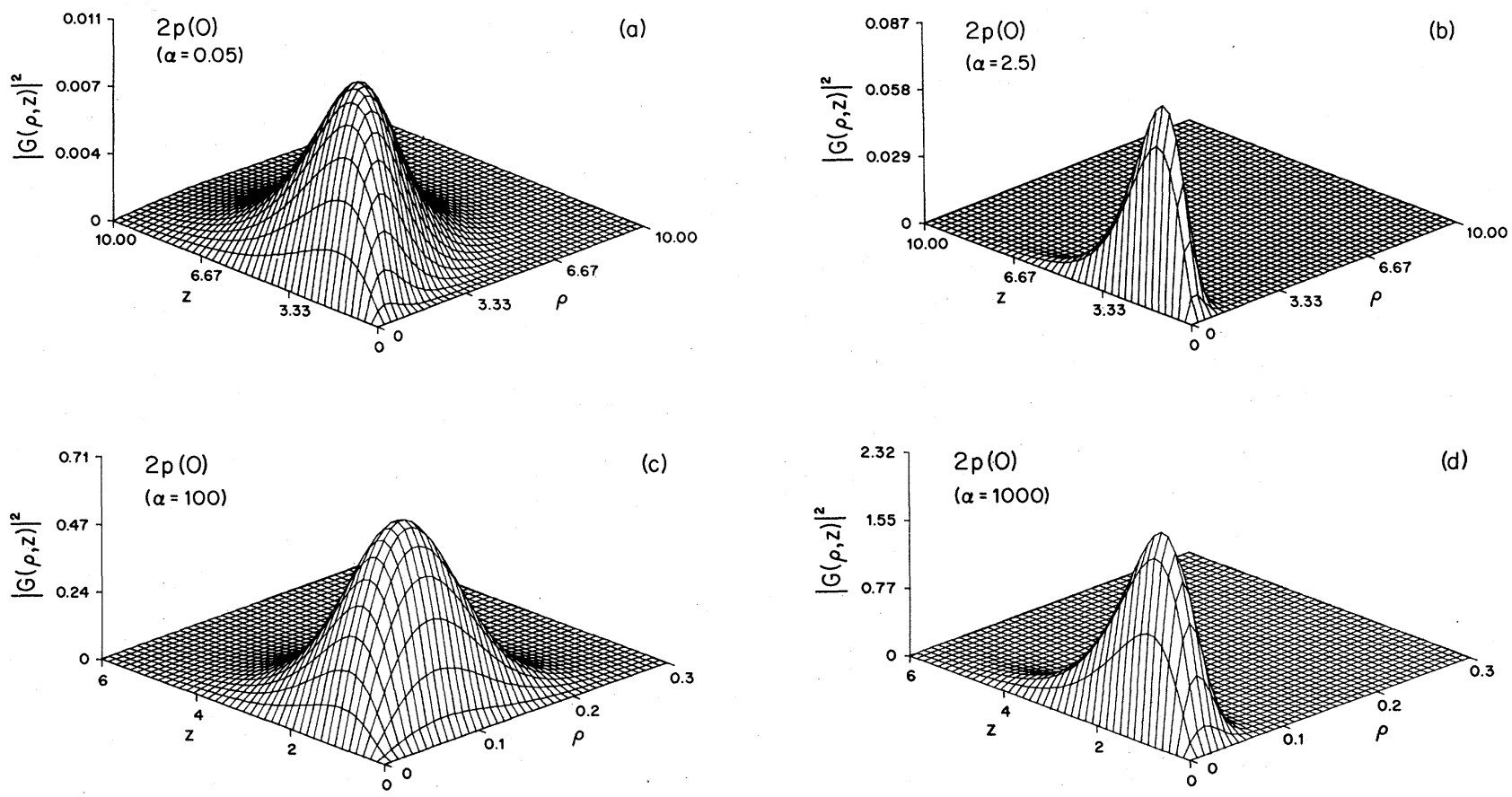

FIG. 6. Absolute square of the adiabatic approximation to the probability amplitude $G(\rho, z)$ [cf. Eq. (19)] for $n m v \pi \equiv 101-$ corresponding to the $2 p\left(m_{l}=0\right)$ level of hydrogen for four values of the magnetic field strength parameter $\alpha$ [cf. Eq. (2)]. 

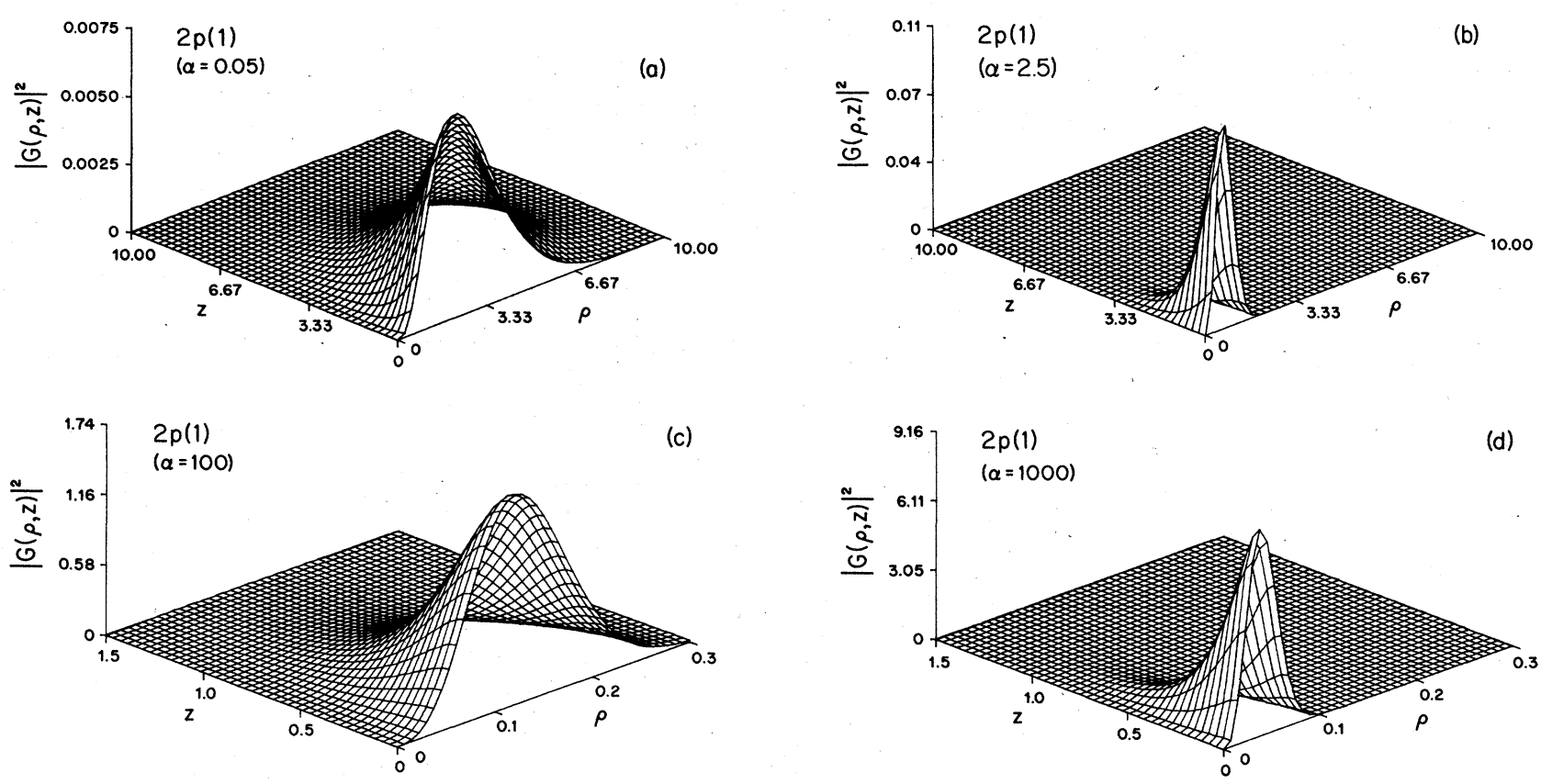

FIG. 7. Absolute square of the adiabatic approximation to the probability amplitude $G(\rho, z)$ [cf. Eq. (19)] for $n m v \pi \equiv 111+$ corresponding to the $2 p\left(m_{l}= \pm 1\right)$ level of hydrogen for four values of the magnetic field strength parameter $\alpha$ [cf. Eq. (2)].

The binding energy of the $v$ th eigenstate for fixed $n, m$, and $\pi$ is thus given by

$$
I_{n m v \pi} \equiv \alpha(n+|m|+m)-E_{v}
$$

For the $s$ and $p$ levels considered in this paper, the values of the threshold shift $\alpha(n+|m|+m)$ are presented in Table I. By subtracting our calculated lower (upper) bounds for the energies $E_{v}$ from the threshold shift, we obtain rigorous upper (lower) bounds on the binding energies $I_{n m v \pi^{*}}$ Note that the binding energies of the $2 p m_{l}= \pm 1$ levels are degenerate since the difference, $2 \alpha$,

TABLE II. Binding energy (in a.u.) of the hydrogen ground state in a uniform magnetic field.

\begin{tabular}{|c|c|c|c|c|c|}
\hline \multirow{2}{*}{$\begin{array}{c}\alpha^{\mathrm{a}} \\
\text { (a.u.) }\end{array}$} & \multirow{2}{*}{$\begin{array}{c}B \\
\left(10^{9} \mathrm{G}\right)\end{array}$} & \multicolumn{2}{|c|}{$\begin{array}{c}\text { Spherical (in italics) and } \\
\text { present cylindrical (in roman) } \\
\text { adiabatic results }\end{array}$} & \multirow{2}{*}{$\begin{array}{l}\text { Variational } \\
\text { lower } \\
\text { bound }\end{array}$} & \multirow{2}{*}{$\begin{array}{l}\text { Eigenfunction } \\
\text { expansion } \\
\text { results } \\
\text { of } \mathrm{RWHR}^{\mathrm{g}}\end{array}$} \\
\hline & & Upper bound & Lower bound & & \\
\hline 0.05 & 0.235 & $0.5475^{b}$ & $0.5474^{b}$ & $0.54753^{c}$ & 0.547527 \\
\hline 0.15 & 0.705 & $0.6296^{\mathrm{b}}$ & $0.6280^{\mathrm{b}}$ & $0.62918^{d}$ & $0.629187(4)$ \\
\hline 0.20 & 0.940 & $0.6657^{b}$ & $0.6622^{b}$ & $0.66438^{\mathrm{e}}$ & $0.664606(4)$ \\
\hline 0.30 & 1.41 & $0.7307^{b}$ & $0.7217^{b}$ & $0.72687^{\mathrm{e}}$ & 0.727463 \\
\hline 0.50 & 2.35 & $0.8418^{\mathrm{b}}$ & $0.8167^{\mathrm{b}}$ & $0.83117^{c}$ & 0.831169 \\
\hline 1.00 & 4.70 & 1.0600 & 0.9832 & $1.02223^{c}$ & 1.022214 \\
\hline 1.50 & 7.05 & 1.1982 & 1.1006 & $1.16453^{c}$ & 1.164533 (11) \\
\hline 2.50 & 11.8 & 1.4094 & 1.2682 & $1.38037^{\mathrm{c}}$ & \\
\hline 12.5 & 58.8 & 2.4087 & 2.2982 & $2.38883^{f}$ & \\
\hline 50.0 & 235.0 & 3.8027 & 3.7360 & $3.78662^{f}$ & $3.790(12)$ \\
\hline 100.0 & 470.0 & 4.7387 & 4.6462 & $4.72251^{\mathrm{f}}$ & 4.727 (12) \\
\hline 1000.0 & 4700.0 & 9.3102 & 9.2754 & $9.28399^{f}$ & $9.305(12)$ \\
\hline
\end{tabular}

${ }^{\mathrm{a}} \alpha=\left(2.12715 \times 10^{-10}\right.$ a.u./G) $B(G)$.

${ }^{\text {b} F r o m ~ A . ~ F . ~ S t a r a c e ~ a n d ~ G . ~ L . ~ W e b s t e r, ~ R e f . ~ 25, ~ T a b l e ~ I I I . ~}$



${ }^{\text {d}}$ From D. M. Larsen, J. Phys. Chem. Solids 29, 271 (1968).

${ }^{\text {e}}$ From E. P. Pokatilov and M. M. Rusanov, Fiz. Tverd. Tela (Leningrad) 10, 3117 (1968) [Sov. Phys.-Solid State 10, 2458 (1969)].

${ }^{\text {f}}$ From P. C. Rech, M. R. Gallas, and J. A. C. Gallas, Ref. 24.

gFrom W. Rösner, G. Wunner, H. Herold, and H. Ruder (RWHR), Ref. 16. The number of expansion terms for each value of the magnetic field is shown in parentheses following the binding energy. 
TABLE III. Binding energy (in a.u.) of the hydrogen $2 p\left(m_{l}=0\right)$ state in a uniform magnetic field.

\begin{tabular}{|c|c|c|c|c|c|}
\hline \multirow{2}{*}{$\begin{array}{c}\alpha^{\mathrm{a}} \\
\text { (a.u.) }\end{array}$} & \multirow{2}{*}{$\begin{array}{c}B \\
\left(10^{9} \mathrm{G}\right)\end{array}$} & \multicolumn{2}{|c|}{$\begin{array}{l}\text { Spherical }{ }^{\mathrm{b}} \text { (in italics) and } \\
\text { present cylindrical (in roman) } \\
\text { adiabatic results }\end{array}$} & \multirow{2}{*}{$\begin{array}{l}\text { Variational } \\
\text { lower } \\
\text { bound }\end{array}$} & \multirow{2}{*}{$\begin{array}{l}\text { Eigenfunction } \\
\text { expansion } \\
\text { results } \\
\text { of } \mathrm{RWHR}^{\mathrm{e}}\end{array}$} \\
\hline & & Upper bound & Lower bound & & \\
\hline 0.05 & 0.235 & 0.1629 & 0.1524 & $0.16238^{c}$ & $0.162410(6)$ \\
\hline 0.5 & 2.35 & 0.2622 & 0.2557 & $0.2596^{c}$ & 0.260007 \\
\hline 1.0 & 4.70 & 0.2991 & 0.2950 & $0.296^{\mathrm{d}}$ & 0.297711 \\
\hline 1.5 & 7.05 & 0.3210 & 0.3181 & & $0.320040(24)$ \\
\hline 2.5 & 11.8 & 0.3483 & 0.3464 & & \\
\hline 12.5 & 58.8 & 0.4223 & 0.4219 & & \\
\hline 50.0 & 235.0 & 0.4638 & 0.4637 & & 0.463618 \\
\hline 100.0 & 470.0 & 0.4775 & 0.4774 & & $0.476532(12)$ \\
\hline 1000.0 & 4700.0 & 0.4956 & 0.4956 & & 0.495595 (12) \\
\hline
\end{tabular}

${ }^{\mathrm{a}} \alpha=\left(2.12715 \times 10^{-10}\right.$ a.u./G) $B(G)$.

${ }^{b}$ From A. F. Starace and G. L. Webster, Ref. 25, Table IV.

'From D. M. Larsen, J. Phys. Chem. Solids 29, 271 (1968).

${ }^{\mathrm{d}}$ From R. R. dos Santos and H. S. Brandi, Phys. Rev. A 13, 1970 (1976).

${ }^{\mathrm{e}}$ From W. Rösner, G. Wunner, H. Herold, and H. Ruder, Ref. 16. The number of expansion terms for each value of the magnetic field is shown in parentheses following the binding energy.

in the threshold shifts exactly cancels the difference of the same amount in the energy levels.

We present our cylindrical adiabatic results for the binding energies corresponding to the $1 \mathrm{~s}, 2 p\left(m_{l}=0\right)$, $2 p\left(m_{l}= \pm 1\right)$, and $2 s$ states of hydrogen in Tables II-V. For the $1 s$, and $2 p$ states our results are rigorous upper and lower bounds on the true binding energy. In order to be most useful to future researchers, we have, firstly, combined our present cylindrical adiabatic results with the previous spherical adiabatic results of Starace and Webster; ${ }^{25}$ only the best available adiabatic upper and lower bounds are shown. Secondly, we have compared our results to the best available variational lower bounds on the binding energies that are known to us whenever these are superior to our adiabatic lower bounds. Lastly we have compared these rigorous upper and lower adiabatic and variational bounds to the very detailed eigenfunction expansion results of Rösner et al. ${ }^{16}$
The binding energies for the $1 \mathrm{~s}$ level are shown in Table II. One sees that the cylindrical adiabatic bounds become superior to the spherical adiabatic bounds ${ }^{25}$ for magnetic field strength parameters $\alpha$ on the order of unity. The rather large difference between the cylindrical upper and lower bounds (on the order of $0.1 \mathrm{a} . \mathrm{u}$.) is due to the poor representation of the $s$ state near $\rho=z=0$ by cylindrically symmetric wave functions. This poor representation results in rather large diagonal coupling matrix elements $\left(f_{n m}, \partial^{2} f_{n m} / \partial z^{2}\right)$. Note, however, that these matrix elements do not enter into the calculation of our upper bounds on the $1 s$ binding energies. When these upper bounds are combined with the best available variational lower bounds, then the differences between the bounds never exceeds about 0.03 a.u. The eigenfunction expansion results of Rösner et al. ${ }^{16}$ are consistent with the upper and lower bounds presented in all cases except possibly a very slight discrepancy with a variational lower-

TABLE IV. Binding energy (in a.u.) of the hydrogen $2 p\left(m_{l}= \pm 1\right)$ state in a uniform magnetic field.

\begin{tabular}{|c|c|c|c|c|c|}
\hline \multirow{2}{*}{$\begin{array}{c}\alpha^{\mathrm{a}} \\
(\text { a.u. })\end{array}$} & \multirow{2}{*}{$\begin{array}{c}B \\
\left(10^{9} \mathrm{G}\right)\end{array}$} & \multicolumn{2}{|c|}{$\begin{array}{l}\text { Spherical }{ }^{\mathrm{b}} \text { (in italics) and } \\
\text { present cylindrical (in roman) } \\
\text { adiabatic results }\end{array}$} & \multirow{2}{*}{$\begin{array}{c}\text { Variational } \\
\text { lower } \\
\text { bound }\end{array}$} & \multirow{2}{*}{$\begin{array}{c}\text { Eigenfunction } \\
\text { expansion } \\
\text { results } \\
\text { of } \mathrm{RWHR}^{\mathrm{e}}\end{array}$} \\
\hline & & Upper bound & Lower bound & & \\
\hline 0.05 & 0.235 & 0.2013 & 0.1882 & $0.20081^{c}$ & $0.200846(5)$ \\
\hline 0.5 & 2.35 & 0.4595 & 0.4524 & $0.456^{c}$ & 0.456597 (13) \\
\hline 1.0 & 4.70 & 0.6021 & 0.5964 & $0.5977^{\mathrm{d}}$ & 0.599613 (15) \\
\hline 1.5 & 7.05 & 0.7059 & 0.7008 & $0.702^{\mathrm{d}}$ & 0.703547 (17) \\
\hline 2.5 & 11.8 & 0.8619 & 0.8575 & $0.86^{\mathrm{c}}$ & \\
\hline 50.0 & 235.0 & 2.6360 & 2.6338 & & 2.6348 \\
\hline 100.0 & 470.0 & 3.3483 & 3.3464 & & $3.3471(12)$ \\
\hline 1000.0 & 4700.0 & 6.9523 & 6.9513 & & $6.9520(12)$ \\
\hline
\end{tabular}

${ }^{\mathrm{a}} \alpha=\left(2.12715 \times 10^{-10}\right.$ a.u. $\left./ \mathrm{G}\right) B(G)$.

${ }^{b}$ From A. F. Starace and G. L. Webster, Ref. 25, Table V.

${ }^{c}$ From D. M. Larsen, J. Phys. Chem. Solids 29, 271 (1968).

${ }^{\mathrm{d}}$ From R. R. dos Santos and H. S. Brandi, Phys. Rev. A 13, 1970 (1976).

${ }^{\mathrm{e}}$ From W. Rösner, G. Wunner, H. Herold, and H. Ruder, Ref. 16. The number of expansion terms for each value of the magnetic field is shown in parentheses following the binding energy. 
TABLE V. Binding energy (in a.u.) of the hydrogen $2 s$ state in a uniform magnetic field.

\begin{tabular}{|c|c|c|c|c|c|}
\hline \multirow{2}{*}{$\begin{array}{c}\alpha^{\mathrm{a}} \\
\text { (a.u.) }\end{array}$} & \multirow{2}{*}{$\begin{array}{c}B \\
\left(10^{9} \mathrm{G}\right)\end{array}$} & \multicolumn{2}{|c|}{$\begin{array}{l}\text { Spherical }{ }^{\mathrm{b}} \text { (in italics) and } \\
\text { present cylindrical (in roman) } \\
\text { adiabatic results }\end{array}$} & \multirow{2}{*}{$\begin{array}{c}\text { Variational } \\
\text { lower } \\
\text { bound }\end{array}$} & \multirow{2}{*}{$\begin{array}{l}\text { Eigenfunction } \\
\text { expansion } \\
\text { results } \\
\text { of } \mathrm{RWHR}^{\mathrm{f}}\end{array}$} \\
\hline & & Upper $^{\mathrm{c}}$ & Lower ${ }^{c}$ & & \\
\hline 0.05 & 0.235 & 0.1503 & 0.0873 & $0.14807^{d}$ & $0.148089(7)$ \\
\hline 0.5 & 2.35 & 0.1594 & 0.1357 & $0.1584^{\mathrm{e}}$ & 0.160469 \\
\hline 1.0 & 4.70 & 0.1744 & 0.1574 & & $0.173944(24)$ \\
\hline 1.5 & 7.05 & 0.1832 & 0.1702 & & 0.18259 \\
\hline 2.5 & 11.8 & 0.1945 & 0.1853 & & \\
\hline 12.5 & 58.8 & 0.2289 & 0.2262 & & \\
\hline 50.0 & 235.0 & 0.2565 & 0.2555 & & 0.25619 \\
\hline 100.0 & 470.0 & 0.2700 & 0.2690 & & 0.26897 \\
\hline 1000.0 & 4700.0 & 0.3063 & 0.3061 & & $0.30624(12)$ \\
\hline
\end{tabular}

${ }^{\mathrm{a}} \alpha=\left(2.12715 \times 10^{-10}\right.$ a.u. $\left./ \mathrm{G}\right) B(G)$.

${ }^{b}$ From A. F. Starace and G. L. Webster, Ref. 25, Table VI.

'The terms "upper" and "lower" here serve solely to distinguish our results obtained, respectively, without any coupling matrix elements and with only the diagonal coupling matrix element included. Since the $2 \mathrm{~s}$ level is the second lowest $m=0$ even parity state, our results are not rigorous upper and lower bounds on the $2 s$ level energy.

${ }^{\mathrm{d}}$ From D. M. Larsen, J. Phys. Chem. Solids 29, 271 (1968).

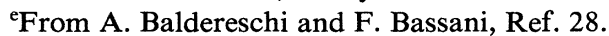

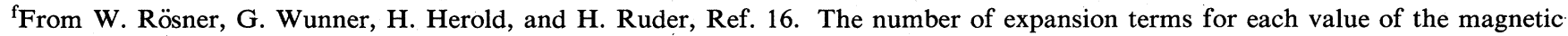
field is shown in parentheses following the binding energy.

bound result for $\alpha=1$.

Our binding energies for the $2 p\left(m_{l}=0\right)$ and $2 p\left(m_{l}= \pm 1\right)$ results are presented in Tables III and IV. Only for $\alpha=0.05$ are the spherical adiabatic results ${ }^{25}$ superior to our cylindrical adiabatic results. Furthermore, for $\alpha \geqq 12.5$ the difference between our upper and lower bounds never exceeds about $4 \times 10^{-4}$ a.u. in the $2 p\left(m_{l}=0\right)$ case and $2 \times 10^{-3}$ a.u. in the $2 p\left(m_{l}= \pm 1\right)$ case. This great improvement from the $1 s$ case stems from the weaker influence of the diagonal coupling matrix element on the lower bound results; for the $m_{l}=0$ case, the function $q_{101-}(z)$ is zero at $z=0$, where the coupling matrix element is largest; for the $m_{l}=1$ case, the radial function $f_{11}(z ; \rho)$ is kept away from the $z$ axis (where $\rho=0$ ) by the centrifugal term in Eq. (7). For values of $\alpha$ greater than about unity, there are no variational lowerbound results known to us which improve upon our own adiabatic lower bounds. Indeed, in the $2 p\left(m_{l}=0\right)$ case our upper and lower bounds are nearly identical (up to our order of accuracy, $10^{-4}$ a.u.) Whereas the eigenfunction expansion results of Rösner et al. ${ }^{16}$ lie between our bounds for all magnetic field strengths in the $2 p\left(m_{l}= \pm 1\right)$ case, they lie slightly outside our bounds for $\alpha=50$ and 100 in the $2 p\left(m_{l}=0\right)$ case. For $\alpha=1000$ they agree exactly with our identical upper and lower bounds to the $2 p\left(m_{l}=0\right)$ binding energy. Note, however, that our results are obtained with a single "configuration" wave function, whereas Rösner et al. employ 12 or more configurations. That we are able to do as well as they is due in large part to the inclusion of the Coulomb field potential in the calculation of our radial functions and to the resulting parametric $z$ dependence of our functions.

Our results for the $2 s$ binding energy are presented in Table V. Since this state is not the lowest one of its symmetry, our results obtained from Eqs. (17) and (18) are not rigorous bounds on the system's energy. We nevertheless label our computed binding energies as "upper" and "lower" to identify the equations used to obtain them. We see once again that the spherical adiabatic results are to be preferred only for $\alpha \leqq 0.05$. Our upper and lower results differ by no more than $3 \times 10^{-3}$ a.u. for $\alpha \geqq 12.5$. Furthermore, for $\alpha \geqq 1$, the eigenfunction expansion results of Rösner et al. ${ }^{16}$ lie between our upper and lower binding energies. Variational lower-bound results are given for $\alpha=0.05$ and 0.5 . Each of these lies between our upper and lower results.

\section{Conclusions}

We conclude that the adiabatic approximation in cylindrical coordinates is an efficient procedure for producing reliable upper and lower bounds for discrete energies of hydrogenic systems, especially for excited levels. When combined with the best available variational lower bounds on the binding energy, in those cases where these are superior (i.e., for $\alpha$ values of the order of unity and for the $1 s$ ground state), the upper and lower bounds presented here provide a stringent test of past and future calculational efforts. Except for a very few isolated instances, our upper and lower bounds are consistent with the eigenfunction expansion results of Rösner et al. ${ }^{16}$ The wave functions and probability densities presented here graphically show clearly the magnetic-field-induced deformations of atomic states.

\section{ACKNOWLEDGMENTS}

This material is based upon work supported by the National Science Foundation under Grant No. PHY8305739. We thank Dr. G. Wunner for bringing Ref. 29 to our attention. 
${ }^{1}$ R. H. Garstang, Rep. Prog. Phys. 40, 105 (1977).

${ }^{2}$ G. Wunner and H. Ruder, J. Phys. (Paris) Colloq. 43, C2-137 (1982).

3J. C. Gay, in Photophysics and Photochemistry in the Vacuum Ultraviolet, edited by S. P. McGlynn, G. L. Findley, and R. H. Huebner (Reidel, Dordrecht, 1985), pp. 631-705.

${ }^{4}$ C. W. Clark, K. T. Lu, and A. F. Starace, in Progress in Atomic Spectroscopy, Part C, edited by H. J. Beyer and H. Kleinpoppen (Plenum, New York, 1984), pp. 247-320.

5J. Simola and J. Virtamo, J. Phys. B 11, 3309 (1978).

${ }^{6}$ J. Killingbeck, J. Phys. B 12, 25 (1979).

7J. E. Avron, I. W. Herbst, and B. Simon, Phys. Rev. A 20, 2287 (1979).

${ }^{8}$ S. C. Kanavi and S. H. Patil, Phys. Lett. A 75, 189 (1980).

${ }^{9}$ V. B. Pavlov-Verevkin and B. I. Zhilinskii, Phys. Lett. A 75, 279 (1980).

${ }^{10}$ H. Ruder, G. Wunner, H. Herold, and M. Reinecke, J. Phys. B 14, L45 (1981).

${ }^{11}$ G. Wunner, H. Ruder, and H. Herold, Phys. Lett. A 85, 430 (1981).

${ }^{12}$ C. M. Bender, L. D. Mlodinow, and N. Papanicolaou, Phys. Rev. A 25, 1305 (1982).

${ }^{13}$ G. Wunner, W. Rösner, H. Ruder, and H. Herold, Astrophys. J. 262, 407 (1982).

14J. C. Le Guillou and J. Zinn-Justin, Ann. Phys. (N.Y.) 147, 57 (1983).

15J. N. Siverman, Phys. Rev. A 28, 498 (1983).

${ }^{16}$ W. Rösner, G. Wunner, H. Herold, and H. Ruder, J. Phys. B 17, 29 (1984).

17J. R. Silva and S. Canuto, Phys. Lett. A 101, 326 (1984).

${ }^{18}$ D. Wintgen and H. Friedrich, J. Phys. B 19, 991 (1986).

19J. M. Wadehra, Astrophys. J. 226, 372 (1978); 248, 874 (1981).

${ }^{20} \mathrm{M}$. Cohen and G. Herman, J. Phys. B 14, 2761 (1981).

21J. Čížek and E. R. Vrscay, Int. J. Quantum. Chem. 21, 27
(1982).

${ }^{22}$ G. A. Arteca, F. M. Fernández, and E. A. Castro, Z. Phys. A 315, 255 (1984).

23J. A. C. Gallas, J. Phys. B 18, 2199 (1985).

${ }^{24}$ P. C. Rech, M. R. Gallas, and J. A. C. Gallas, J. Phys. B 19, L215 (1986).

${ }^{25}$ A. F. Starace and G. L. Webster, Phys. Rev. A 19, 1629 (1979).

${ }^{26}$ L. I. Schiff and H. Snyder, Phys. Rev. 55, 59 (1939).

${ }^{27}$ R. J. Elliott and R. Loudon, J. Phys. Chem. Solids 15, 196 (1960).

${ }^{28}$ A. Baldereschi and F. Bassani, in Proceedings of the 10th International Conference on the Physics of Semiconductors, Cambridge, Mass., AEC Oak Ridge Conf. No. 700801, edited by S. P. Keller, J. C. Hensel, and F. Stern (U.S. A.E.C., Washington, D.C., 1970), pp. 191-196.

${ }^{29}$ K. Tanaka and M. Shinada, J. Phys. Soc. Jpn. 34, 108 (1973).

${ }^{30}$ V. Canuto and D. C. Kelly, Astrophys. Space Sci. 17, 277 (1972).

${ }^{31}$ G. Wunner, Astrophys. J. 240, 971 (1980).

${ }^{32}$ C. R. Liu and A. F. Starace, Bull. Am. Phys. Soc. 30, 873 (1985).

${ }^{33}$ L. D. Landau and E. M. Lifshitz, Quantum Mechanics, 2nd ed., translated by J. B. Sykes and J. S. Bell (Pergamon, Oxford, 1965), p. 426.

${ }^{34}$ G. L. Surmelian and R. F. O’Connell, Astrophys. J. 190, 741 (1974).

${ }^{35}$ V. B. Pavlov-Verevkin and B. I. Zhilinskii, Phys. Lett. A 78, 244 (1980).

${ }^{36}$ See the Appendix of Ref. 25. The proofs presented there for the adiabatic approximations in spherical coordinates carry over straightforwardly to the cylindrical coordinate cases presented here. 